$\xi_{p}=$

\title{
Fuzzy Generalized Fractal Dimensions Using Inter-Heartbeat Interval Dynamics in ECG Signals for Age Related Discrimination
}

\author{
D. Easwaramoorthy ${ }^{1, *}$, P.S. Eliahim Jeevaraj ${ }^{2}$, A. Gowrisankar ${ }^{3}$, A. Manimaran ${ }^{4}$ and S. Nandhini ${ }^{5}$ \\ 1,3,4,5 Department of Mathematics, School of Advanced Sciences (SAS), Vellore Institute of Technology (VIT), \\ Vellore - 632 014, Tamil Nadu, India. \\ ${ }^{2}$ Department of Computer Science, Bishop Heber College, Tiruchirappalli - 620 017, Tamil Nadu, India. \\ *Corresponding Author.E mail: easandk@gmail.com
}

\begin{abstract}
Fractal theory is the propelled technique to analyze the non-linear signals with more complexity. Quantification of chaotic nature and complexity of the multifaceted therapeutic signals requires the estimation of the spectrum of Generalized Fractal Dimensions (GFD) where the complexity means greater inconstancy in the general form of fractal dimension range. This paper has proposed a fuzzy multifractal technique to analyze the age related classifications by using the Fuzzy Generalized Fractal Dimensions (F-GFD) with Gaussian fuzzy valued function through the cardiac inter-beat interval dynamics in electrocardiogram (ECG) signals. It has been revealed that, the designed Fuzzy GFD method accurately categorizes the young and old age subjects by graphical comparison with the typical GFD method. The classification rate of young and elderly subjects has also supported statistically by ANOVA test. Hence the fuzzified multifractal analysis accomplishes significantly to discriminate age groups than the classical multifractal analysis in heartbeat rate time series from ECG signals and also the conventional GFD is a specific case of the proposed F-GFD.
\end{abstract}

Keywords: Fractal Analysis, Generalized Fractal Dimensions, Fuzzy Membership Functions, Electrocardiogram Signals, Heartbeat Time Series.

\section{Introduction}

In Mathematics, the classical Euclidean geometry deals with sets existing in whole number measurements, though fractal geometry manages protests in non-whole number measurements. A Fractal is commonly an irregular or divided geometric shape which can be break up into fragments, every one of which is (at any rate roughly) a decreased size duplicate of the entire, a property called selfsimilarity. The terminlogy Fractal has instituted by Benoit Mandelbrot, the Professor Emeritus, in his key exposition which has gotten from the Latin literature, fractus meaning that broken, cracked or fractured to portray sets that were excessively sporadic, making it impossible to match into an ordinary geometrical form. In his unique article, Mandelbrot scientifically characterized a Fractal as a set with the Hausdorff dimension is strictly greater than its topological dimension [1,11]. Generally, a fractal set is a set that is more unpredictable than the sets considered in traditional geometry. Karl Weierstrass provided a function with the noninstinctive condition of being continuous at everywhere but differentiable at nowhere, called Weierstrass Function, whose graph would today be reflected as fractal $[1,6,11]$. The intricacy and anomaly that can be normally found in numerous physical and biological non-linear frameworks; and which has been investigated by the strategies of fractal analysis and processed by the nonwhole number or fragmentary measure called Fractal Dimension. In the study of experimental systems, the correlation dimension measurement is more feasible among all the non-linear methods. Since, the magnitude of the calculated correlation dimension is a single value from the class of fractal dimension spectrum; general- ly it does not indicate the intricacy of the data. This single scalar value is inadequate to exhibit the inconsistent nature of the chaotic signals. Such a chaotic object which is not homogenous is called Multi fractal and is analyzed by GFD. There is an advantage by using the generalized form of fractal dimensions rather than using only some of the dimensions $[7,8,10]$. The complexity of the time series with nonlinearity has been inspected under different settings by the multi-fractal measure called GFD [2, 3, 4, 5, 16, 19]. Afterward, GFD has characterized for noisy images to analyze the rate of intricacy $[17,18]$. Additionally, F-GFD has been generalized from the traditional GFD for assessing the chaotic nature of the mathematical waveforms created by Weierstrass Functions [20].

Electrocardiogram (ECG) is the essential sign most generally utilized in the clinical condition. It gives knowledge into the understanding of many age related cardiac complaints [9]. The tremendous existing literature on automatic ECG classification involves lot more strategies dependent on pattern recognition methodologies, artificial neural networks, support vector machines, linear discriminant analysis, clustering techniques and other soft computing methods have been investigated by different analysts $[12,14]$. In all physical diagnosis, the physicians are perceived the diseases based on the age group of the patients. At the outset of diagnosing process, the age can be discriminated through the ECG by interheartbeat interval dynamics.

A fuzzy multifractal measure for biomedical signals to distinguish the age group of subjects has been presented in this research work. The proposed method discriminates the young and elderly subjects by applying the F-GFD with Gaussian fuzzy function in ECG Signals through the cardiac inter-heartbeat time interval dynamics. 
The structure of the paper is as presented as: in Section 2, Renyi entropy measure and GFD Measure for experimental signals are depicted. Additionally, Fuzzy Renyi entropy measure and F-GFD measure for signals are characterized. In Section 3 the ECG Data has been analyzed. The acquired computational outcomes are examined clearly in Section 4. At long last, finishing up comments is introduced in Section 5.

\section{Mathematical Methods}

\subsection{Renyi Entropy}

Renyi entropy [13, 15] assumes a noteworthy record in the information theory. Renyi entropy, a generality of Shannon entropy, is one of the group of functionals for evaluating the diversity, vagueness or arbitrariness of a given framework. It was presented by Alfred Renyi [13]. Renyi entropy is otherwise called generalized entropy of a given probability measures.

For the given probability distribution and a real number $q$ such that $q \geq 0$ and $q \neq 1$, the Renyi Entropy with the order $q$ is described as below.

$$
S_{q}=\frac{1}{1-q} \log _{2}\left(\sum_{i=1}^{N} p_{i}^{q}\right)
$$

where $p_{i} \in[0,1]$ is the probability values associated correspondingly to each $x_{\mathrm{i}}$ for $i=1,2, \ldots, N$ taken by the random variable.

\subsection{Fuzzy Renyi Entropy}

The Fuzzy Renyi Entropy of order $q$, provided $q$ is greater than or equal to 0 and $q$ is not equal to 1 on a given set $\mathrm{S}$ is given below

$F R E_{q}=\frac{1}{1-q} \log _{2}\left(\sum_{i=1}^{N}\left(\sum_{x \in S_{i}} \mu(x)\right)^{q}\right)$

where $\mu: S \rightarrow[0,1]$ is the fuzzy logic membership function over the underlying space $S$ consists of $N$ number of partitions, such as $S_{1}, S_{2}, \ldots, S_{\mathrm{N}}$.

\subsection{Multifractal Analysis}

The Renyi entropies plays a major role in non-linear and statistical analysis since they are characterized by irregularity and haphazardness. These entropies result in a range of indices of fractal measure called as GFD. In 1983, Multifractal theory was developed based on GFD measure [7, 8]. The GFD process for noisy signals is discussed in this section $[2,3,4,5,7,8,16,19]$.

\subsubsection{GFD for Chaotic Signals}

In order to define the GFD measure, the required probability distribution of a given multifaceted signal is constructed as below: The entire array of the Signal Time Series is separated into $N$ intervals (bins) as follows

$N=\frac{V_{\max }-V_{\min }}{r}$

where $V_{\max }$ and $V_{\min }$ represents the maximum value and the minimum value of the signals obtained by the experiments, respectively; and $r$ is the uncertainty factor, which depends on the device used to register the Signal entries.
Now the probability that the time series passes through the $i^{\text {th }}$ bin of size $r$ is defined as

$p_{i}=\lim _{N \rightarrow \infty} \frac{N_{i}}{N}, \quad i=1,2, \ldots, N$

where $N_{i}$ is the number of times the time series lies on the $i^{\text {th }}$ bin of with $r$ length.

Then, the Renyi Fractal Dimensions or Generalized Fractal Dimensions $(G F D)$ of order $q \in(-\infty, \infty)$ of the given probability measures, denoted by $D_{q}$, is given below

$D_{q}=\lim _{r \rightarrow 0} \frac{1}{q-1} \frac{\log _{2}\left(\sum_{i=1}^{N} p_{i}^{q}\right)}{\log _{2} r}$.

Here GFD measure is defined by means of generalized Renyi Entropy.

\subsection{Fuzziness of Multi-fractal Analysis}

Here F-GFD to quantify the fuzziness of chaotic waveforms.

\subsubsection{F-GFD for Chaotic Signals}

F-GFD for a given fractal waveforms is defined as follows [20].

The whole exhibit of the set $S$ is separated into $N$ number of intervals such as $S_{1}, S_{2}, \ldots, S_{\mathrm{N}}$ as follows :

$N=\frac{V_{\max }-V_{\min }}{r}$

where $V_{\max }$ and $V_{\min }$ symbolizes the supremum and the infimum values of the signals obtained in the experimentations, respectively; and $r$ is the experimental vagueness error, which depends on the instrument used to register the signals.

A fuzzy logic membership function on the set $S$ divided by $N$ number of bins $S_{1}, S_{2}, \ldots, S_{\mathrm{N}}$ is characterized as a function, $\mu: S \rightarrow[0,1]$.

Then, the Fuzzy Generalized Fractal Dimensions ( $F-G F D$ ) with the order $q \in(-\infty, \infty)$ for the given fuzzy logic membership function on the representative signal $S$ is denoted and defined by $F D_{q}$ as below

$F D_{q}=\lim _{r \rightarrow 0} \frac{1}{q-1} \frac{\log _{2}\left(\sum_{i=1}^{N}\left(\sum_{x \in S_{i}} \mu(x)\right)^{q}\right)}{\log _{2} r}$.

Here F-GFD measure is defined by means of generalized Fuzzy Renyi Entropy. It is also known as Fuzzy Renyi Fractal Dimensions.

\subsection{Gaussian Fuzzy Logic Membership Function}

The general Gaussian fuzzy logic membership function, $g: S$ $\rightarrow[0,1]$, on the set $S$ with respect to the mean $(\bar{x})$ and the standard deviation $(\sigma)$ is defined below.

$g(x ; \bar{x}, \sigma)=e^{\frac{-(x-\bar{x})^{2}}{2 \sigma^{2}}}$

\section{Experimental ECG Data}

Two sets of five youthful (21 - 3434 years of age) and 5 people of age (68 - 8134 yrs of age) thoroughly examined normal subjects experienced 2 hours of constant recumbent resting while continuous electrocardiogram (ECG) signals were registered. All were staying in a resting state in sinus mood while continuous electrocardiogram (ECG) signals were collected. All subjects stayed in a resting state in sinus mood while viewing the specific motion pic- 
ture to help look after attentiveness. Every subgroup of subjects incorporates equal numbers of men and women. The continuous ECG was digitized at $250 \mathrm{~Hz}$. Every heartbeat was commented on utilizing an programmed arrhythmia detection algorithm, and each beat annotation was checked by visual review. The $R-R$ interim (inter-heartbeat interval) time arrangement for each subject was then registered [9]. An example of ECG epochs from every one of two age bunches is portrayed in Figure 1.
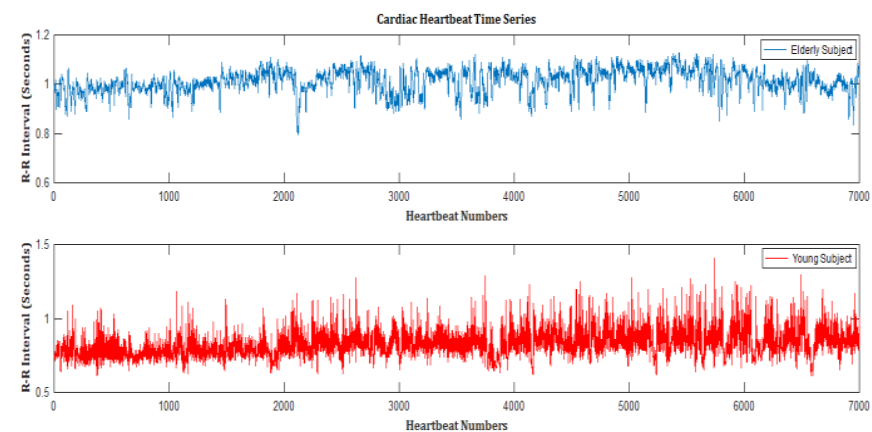

Figure 1: Sample ECG Epochs from Elderly and Young Healthy Subjects

\section{Results and Discussions}

To study the proficiency of the developed Fuzzy Multifractal measure, simulations are performed with the representative biomedical ECG Signals described in Section 3 in MATLAB software.

The probability distributions of each of the $R-R$ interval time series of clinical ECG Data were obtained and the corresponding GFD were also calculated for the same. The fuzzy logic membership function values for each of the demonstrative time series were also determined by means of the Gaussian fuzzy logic membership function $(g)$. From the calculated Gaussian membership values, the F-GFD for all representative signals was determined.

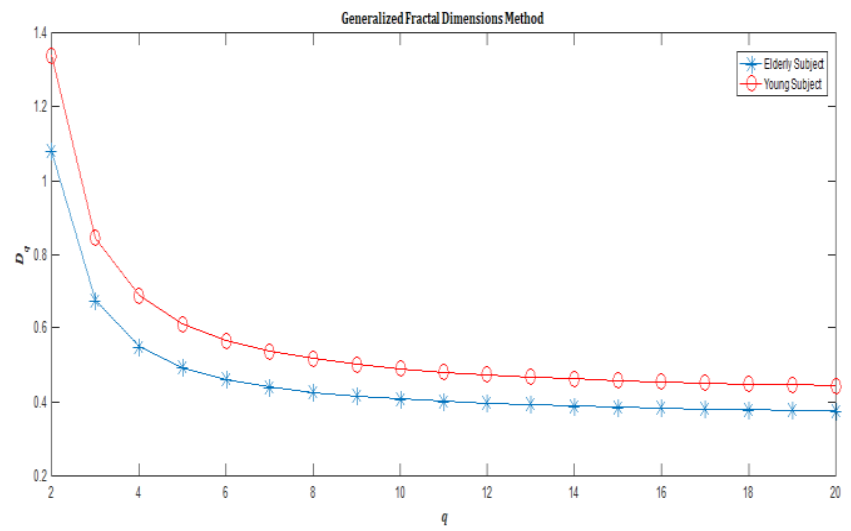

Figure 2: Generalized Fractal Dimensions Spectra for R-R Interval Time Series of Elderly and Young Subjects

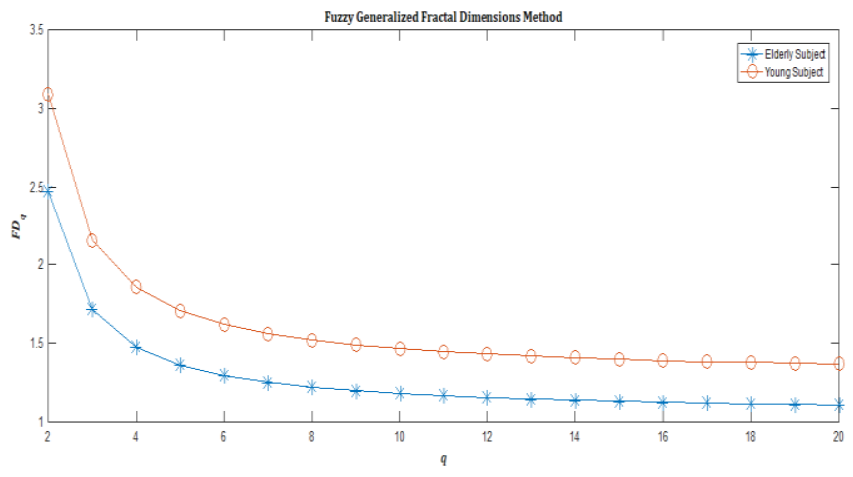

Figure 3: F-GFD Spectra for R-R Interval Time Series of Elderly and Young Subjects
Table 1: One-way ANOVA Tables for GFD and Fuzzy GFD Spectrum of Elderly and Young Subjects

(a). GFD Method

\begin{tabular}{lcrrrr}
\hline Source & SS & df & MS & F & Prob>F \\
Columns & 0.09191 & 1 & 0.09191 & 2.51 & 0.1221 \\
Error & 1.3199 & 36 & 0.03666 & & \\
Total & 1.41182 & 37 & & & \\
\hline
\end{tabular}

\begin{tabular}{lccccr}
\multicolumn{6}{c}{$(b)$. Fuzzy GFD Method } \\
\hline Source & SS & df & MS & F & Prob>F \\
Columns & 0.93736 & 1 & 0.93736 & 6.81 & 0.0131 \\
Error & 4.95819 & 36 & 0.13773 & & \\
Total & 5.89555 & 37 & & & \\
\hline
\end{tabular}

The processed GFD measures are sketched against the corresponding order values $q$ for all demonstrative interval time series as in Figure 2. Correspondingly the calculated F-GFD measures are graphically drawn against the corresponding order values $q$ for a wide range of time arrangement as appeared in Figure 3. To examine the average changes between Elderly and Young ECG time series statistically, ANOVA Tables and Notched Type of Box Plots for GFD and F-GFD measures are constructed for every $R$ $R$ interval time series of experimental ECG Data; and are exposed in Table 1 and Figures $4 \& 5$.

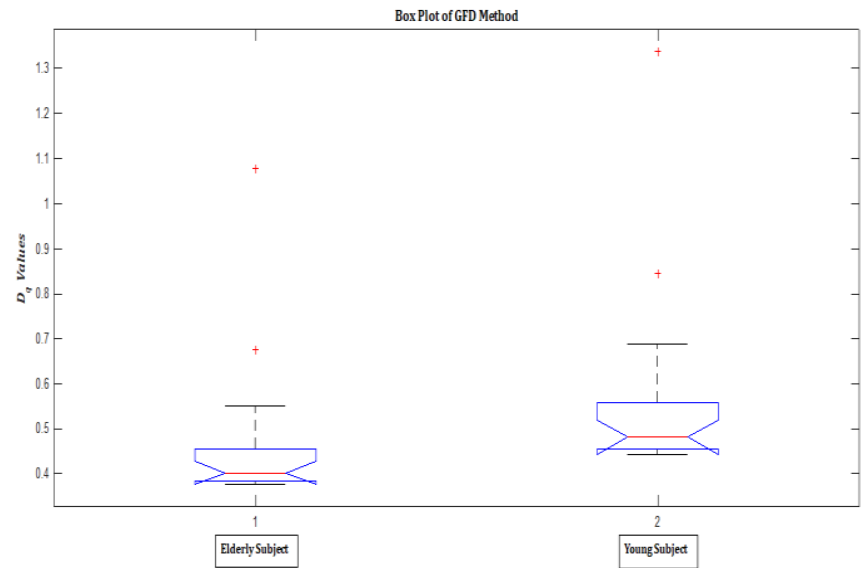

Figure 4: Box Plots for GFD Ranges of Elderly \& Young Subjects

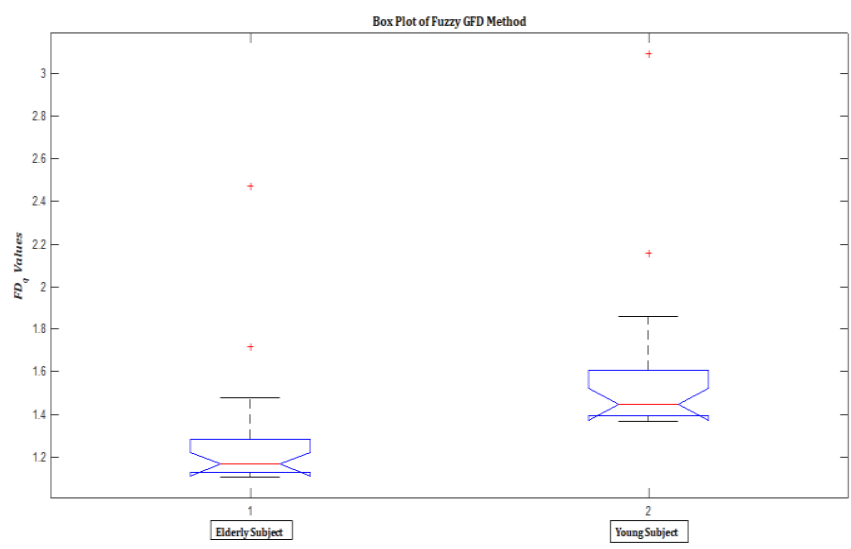

Figure 5: Box Plots for Fuzzy GFD Ranges of Elderly \& Young Subjects

In Figure 3, the Fuzzy GFD spectra of $R-R$ interval time series expressively classified the Elderly and Young Subjects than the GFD spectra of $R-R$ interval time series as in Figure 2. In Table 1, ANOVA Test also underpins our composed strategies factually, than the GFD Method. The box plots for Fuzzy GFD Method in 
Figure 5 demonstrates the distinctions in mean variance than the box plots for GFD Method as in Figure 4.

Therefore, Figures 2, 3, $4 \& 5$ and Table 1 show that the fuzzy multi-fractal analysis has a prominence role in age related discrimination by implementing F-GFD instead of GFD technique.

\section{Conclusion}

This investigation describes a fuzzy multi-fractal theory with the end goal to separate the two age gatherings (youthful and elderly subjects) by utilizing the F-GFD with Gaussian fuzzy membership function in ECG waveforms through the cardiac interheartbeat time series. Further, ANOVA test has been performed which measurably bolstered the order rate between two age gatherings. Accordingly the simulation results demonstrate that the execution of fuzzy multi fractal analysis is significant is huge when contrasted with traditional multi fractal analysis pertain to age related issues.

\section{References}

[1] M.F. Barnsley, Fractals Everywhere, $2^{\text {nd }}$ edn., Academic Press, USA, 1993.

[2] D. Easwaramoorthy and R. Uthayakumar, Estimating the Complexity of Biomedical Signals by Multifractal Analysis, Proceedings of the IEEE Students' Technology Symposium, IEEE Xplore Digital Library, IEEE, USA, (2010) 6-11.

[3] D. Easwaramoorthy and R. Uthayakumar, Analysis of EEG Signals using Advanced Generalized Fractal Dimensions, Proceedings of the Second International Conference on Computing, Communication and Networking Technologies, IEEE Xplore Digital Library, IEEE, USA, (2010) 1-6.

[4] D. Easwaramoorthy and R. Uthayakumar, Analysis of Biomedical EEG Signals using Wavelet Transforms and Multifractal Analysis, Proceedings of the IEEE International Conference on Communication Control and Computing Technologies, IEEE Xplore Digital Library, IEEE, USA, (2010) 544-549.

[5] D. Easwaramoorthy and R. Uthayakumar, Improved Generalized Fractal Dimensions in the Discrimination between Healthy and Epileptic EEG Signals, Journal of Computational Science, 2(1) (2011) 31-38.

[6] K.J. Falconer, Fractal Geometry: Mathematical Foundations and Applications, $2^{\text {nd }}$ edn., John Wiley \& Sons Ltd., England, 2003.

[7] P. Grassberger, Generalized dimensions of strange attractors, Physics Letters A, 97 (1983) 227-320.

[8] H.G.E. Hentschel and I. Procaccia, The Infinite Number of Generalized Dimensions of Fractals and Strange Attractors, Physica 8D, (1983) 435-444.

[9] N. Iyengar, C-K. Peng, R. Morin, A.L. Goldberger and L.A. Lipsitz, Age-related alterations in the fractal scaling of cardiac interbeat interval dynamics, American Journal of Physiology - Regulatory, Integrative and Comparative Physiology, 271(4) (1996) 1078-1084.

[10] M. Lakshmanan and S. Rajasekar, Nonlinear Dynamics: Integrability, Chaos and Patterns, Springer-Verlag Heidelberg, 2003.

[11] B.B. Mandelbrot, The Fractal Geometry of Nature, W.H. Freeman and Company, New York, 1983.

[12] Mario Sansone, Roberta Fusco, Alessandro Pepino and Carlo Sansone, Electrocardiogram Pattern Recognition and Analysis Based on Artificial Neural Networks and Support Vector Machines: A Review, Journal of Healthcare Engineering, 4(4) (2013) 465504.

[13] A. Renyi, On a new axiomatic theory of probability, Acta Mathematica Hungarica, 6 (1955) 285-335.

[14] Rishi Raj Sharma and Ram Bilas Pachori, Baseline wander and power line interference removal from ECG signals using eigenvalue decomposition, Biomedical Signal Processing and Control, 45 (2018) 33-49.

[15] C.E. Shannon, The Mathematical Theory of Communication, University of Illinois Press, Champaign, IL, 1998.

[16] R. Uthayakumar and D. Easwaramoorthy, Multifractal-Wavelet Based Denoising in the Classification of Healthy and Epileptic EEG Signals, Fluctuation and Noise Letters, 11(4) (2012) Article No.: 1250034 (22 Pages).
[17] R. Uthayakumar and D. Easwaramoorthy, Generalized Fractal Dimensions in the Recognition of Noise Free Images, Proceedings of the International Conference on Computing, Communication and Networking Technologies, IEEE Xplore Digital Library, IEEE, USA, (2012) 1-5.

[18] R. Uthayakumar and D. Easwaramoorthy, Multifractal Analysis in Denoising of Color Images, Proceedings of the International Conference on Emerging Trends in Science, Engineering and Technology, IEEE Xplore Digital Library, IEEE, USA, (2012) 228-234.

[19] R. Uthayakumar and D. Easwaramoorthy, Epileptic Seizure Detection in EEG Signals using Multifractal Analysis and Wavelet Transform, Fractals, 21(2) (2013) Article No.: 1350011 (24 Pages).

[20] R. Uthayakumar and D. Easwaramoorthy, Fuzzy Generalized Fractal Dimensions for Chaotic Waveforms, Chaos, Complexity and Leadership 2012, Springer Proceedings in Complexity, (2014) 411422. 